\title{
La conservation des ressources en eau par la mise en oeuvre de paiements pour services environnementaux dans un bassin versant
}

\section{Water resources conservation through the implementation of payments for environmental services in a watershed}

\section{Abdelmohssin El Mokaddem et Fayçal Benchekroun}

Volume 29, numéro 2, 2016

Reçu le 5 juin 2014, accepté le 16 octobre 2014

URI : https://id.erudit.org/iderudit/1036543ar

DOI : https://doi.org/10.7202/1036543ar

Aller au sommaire du numéro

Éditeur(s)

Université du Québec - INRS-Eau, Terre et Environnement (INRS-ETE)

ISSN

1718-8598 (numérique)

Découvrir la revue

Citer cet article

El Mokaddem, A. \& Benchekroun, F. (2016). La conservation des ressources en eau par la mise en oeuvre de paiements pour services environnementaux dans un bassin versant. Revue des sciences de l'eau / Journal of Water Science, 29(2), 109-117. https://doi.org/10.7202/1036543ar

\section{Résumé de l'article}

Les paiements pour services environnementaux (PSE) sont un outil économique d'incitation de plus en plus utilisé pour la conservation des bassins versants. En se basant sur la notion des services environnementaux, il permet de concilier plusieurs objectifs de conservation au niveau des bassins versants pour une finalité d'amélioration des attributs qui caractérisent les ressources en eau. L'amélioration de la disponibilité de l'eau en surface et en sous-sol, ainsi que l'amélioration de sa qualité par la réduction de l'érosion hydrique et la filtration des nitrates, sont des exemples de services qui permettent de renforcer la conservation des bassins versants et d'améliorer l'efficacité économique des investissements. Au Maroc, la conservation des bassins versants a traversé des étapes importantes vers la mise en place d'une gestion intégrée, toutefois les PSE ne sont pas encore mis en oeuvre. Leur introduction au niveau du pays permettra des apports et sera en même temps contrariée par un certain nombre de limites. L'article présente la notion de services environnementaux et les fondements économiques des PSE, puis apporte un point de vue et des propositions quant à leur mise en oeuvre pour améliorer la conservation des bassins versants dans le contexte spécifique du Maroc.
Tous droits réservés @ Revue des sciences de l'eau, 2016

Ce document est protégé par la loi sur le droit d'auteur. L'utilisation des services d’Érudit (y compris la reproduction) est assujettie à sa politique d'utilisation que vous pouvez consulter en ligne.

https://apropos.erudit.org/fr/usagers/politique-dutilisation/ 


\section{LA CONSERVATION DES RESSOURCES EN EAU PAR LA MISE EN GEUVRE DE PAIEMENTS POUR SERVICES ENVIRONNEMENTAUX DANS UN BASSIN VERSANT}

Water resources conservation through the implementation of payments for environmental services in a watershed

\section{ABDELMOHSSIN EL MOKADDEM ${ }^{\prime *}$, FAYÇAL BENCHEKROUN ${ }^{2}$}

${ }^{1}$ Institut Agronomique et Vétérinaire Hassan II, Département des sciences humaines, Madinat AL Irfane, BP 6202, Rabat, Maroc

${ }^{2}$ Institut Agronomique et Vétérinaire Hassan II, Département des ressources naturelles et environnement, Madinat AL Irfane, BP 6202, Rabat, Maroc

Reçu le 5 juin 2014, accepté le 16 octobre 2014

\section{RÉSUMÉ}

Les paiements pour services environnementaux (PSE) sont un outil économique d'incitation de plus en plus utilisé pour la conservation des bassins versants. En se basant sur la notion des services environnementaux, il permet de concilier plusieurs objectifs de conservation au niveau des bassins versants pour une finalité d'amélioration des attributs qui caractérisent les ressources en eau. L'amélioration de la disponibilité de l'eau en surface et en sous-sol, ainsi que l'amélioration de sa qualité par la réduction de l'érosion hydrique et la filtration des nitrates, sont des exemples de services qui permettent de renforcer la conservation des bassins versants et d'améliorer l'efficacité économique des investissements. Au Maroc, la conservation des bassins versants a traversé des étapes importantes vers la mise en place d'une gestion intégrée, toutefois les PSE ne sont pas encore mis en œuvre. Leur introduction au niveau du pays permettra des apports et sera en même temps contrariée par un certain nombre de limites. L'article présente la notion de services environnementaux et les fondements économiques des PSE, puis apporte un point de vue et des propositions quant à leur mise en œuvre pour améliorer la conservation des bassins versants dans le contexte spécifique du Maroc.

Mots-clés : Paiements pour services environnementaux, régulation bydrologique, conservation, bassins versants, Maroc, eau.

\section{ABSTRACT}

Payments for environmental services (PES) are an economic incentive tool increasingly used for watershed conservation. Based on the concept of environmental services, the PES approach allows the combination of several conservation objectives at the watershed scale to improve the 
attributes of the water resources. Improving the availability of surface water and groundwater recharge, and improving water quality by reducing water erosion and enhancing nitrate filtration, are examples of services that enhance the conservation of watersheds and improve the economic efficiency of investments. In Morocco, watershed conservation has gone through important steps towards the implementation of integrated management, but PES are not yet implemented. Their implementation in the country will be of interest and simultaneously thwarted by a number of limitations. This paper presents the concept of environmental services and the economic foundations of PES, then presents a point of view and proposals for their implementation to improve watershed conservation in the specific context of Morocco.

Key Words: Payments for environmental services, hydrological regulation, conservation, watershed, Morocco, water.

\section{INTRODUCTION}

Au Maroc, les enjeux associés à la valorisation des ressources en eau sont multiples. L'eau est un bien économique rare et essentiel pour le fonctionnement de l'économie et pour le bienêtre humain. Son usage nécessite non seulement sa disponibilité en quantités suffisantes, mais aussi un niveau de qualité qui permet d'en bénéficier à moindre coût. Ainsi, conserver l'eau ne signifie pas uniquement bien gérer la demande, mais également, améliorer sa disponibilité et sa qualité.

La modification des écosystèmes par les activités économiques, en fonction de leur nature et des modes de gestion et d'allocation des terres qu'elles adoptent, peut influencer négativement ou positivement la disponibilité et la qualité de l'eau (EROL et RANDHIL, 2013; FIQUEPRON et al., 2013). Toutefois, cette modification donne lieu à des coûts directs et indirects qui peuvent être insupportables et pénalisants. Les efforts fournis en amont produisent des bénéfices au profit d'autres agents économiques qui en bénéficient, en aval, sans rien payer. Cette situation est non efficiente d'un point de vue économique. Pour qu'un changement de comportement en termes de pratiques de gestion ait lieu en faveur dela conservation de l'eau, une des possibilités est de faire payer les bénéficiaires pour financer les coûts qu'implique ce changement.

Plusieurs instruments et mécanismes économiques ont été développés et appliqués, depuis plus de 20 ans, pour pallier ces inefficiences de différentes manières. Les plus populaires sont les outils d'incitation basés sur le marché. Ils adoptent le principe de l'internalisation des externalités et certains d'entre eux se servent d'une approche récente dite " approche par services environnementaux ». Les services environnementaux (SE) sont des bénéfices tirés des écosystèmes par les êtres humains (biodiversité, récréation, eau de bonne qualité, etc.) (MEA, 2005).

Les paiements pour services environnementaux (PSE) ont fait leur apparition dans cette vaste famille d'instruments. Ils jouissent d'une popularité croissante malgré leur apparition récente. Leur adoption pour la conservation des bassins versants connaît une large diffusion aussi bien dans le monde développé que dans les pays en développement. Ils visent la création de marchés où des services environnementaux sont échangés en se servant de l'évaluation économique de ces services.

Au Maroc, la conservation des écosystèmes et des bassins versants en particulier, souffre d'insuffisances qui perpétuent une dégradation croissante des ressources en eau. Les exemples de mise en ouvre des PSE dans plusieurs pays montrent que ce mécanisme offre plusieurs avantages en termes d'incitation à la conservation. La mise en ouvre de PSE dans le contexte marocain soulève plusieurs défis. Notamment, la possibilité de satisfaction des conditions requises pour qu'un PSE soit conforme aux fondements économiques et à la logique commune des PSE. L'aptitude des spécificités du contexte social, politique et économique marocain à permettre une mise en œuvre réussie des PSE est aussi une dimension à considérer.

Le présent article propose de préciser, dans un premier temps, la définition des SE et la présentation d'une typologie. Découvrir, dans un deuxième temps, le concept de PSE, sa logique, ses fondements et un état des lieux synthétique de son application pour la conservation des bassins versants. Dans un troisième temps, nous allons passer en revue le cas du Maroc en discutant du contexte de la conservation des ressources en eau et des bassins versants. En dernière section, nous présentons les apports potentiels des PSE dans le contexte du Maroc, ainsi que leurs limites, et identifions des prérequis pour une mise en œuvre adaptée aux spécificités des bassins versants du pays.

\section{SERVICES ÉCOSYSTÉMIQUES: UNE VALEUR ÉCONOMIQUE ET UNE NOUVELLE VISION POUR LA CONSERVATION DES ÉCOSYSTÈMES}

Les services écosystémiques (SE) correspondent aux " avantages que les sociétés humaines tirent des écosystèmes naturels » (DAILY, 1997). Ils se réfèrent aussi à des « conditions et processus écologiques qui assurent le bien-être humain " (DAILY, 1997; MEA, 2005). Ils se distinguent des processus 
écologiques (flux d'énergie ou de matières) et des propriétés propres aux écosystèmes (stabilité, résilience, etc.) par leur usage en économie et/ou leur contribution à l'amélioration du bien-être humain. L'évaluation du Millénaire (MEA, 2005) propose une classification des services écosystémiques en quatre catégories en fonction des bénéfices auxquels ils correspondent (Tableau 1).

Il est à noter que l'appellation "services écosystémiques " a connu une transition du MEA (2005) au rapport de la FAO (2007) qui a initié l'utilisation de "services environnementaux " pour désigner la même notion.

L'usage des SE, bien qu'il soit une source de bénéfices, reste non visible dans la comptabilité économique. L'idée de l'évaluation économique de la valeur des usages a permis d'associer une nouvelle vision aux écosystèmes. La multifonctionnalité des écosystèmes est désormais la source d'une multitude de bénéfices. Selon cette vision, les forêts, les terres de pâturage et les terres agricoles ne produisent pas seulement du bois, du fourrage ou des produits agricoles, mais fournissent aussi des services de régulation hydrologique, des aménités paysagères, etc.

Pour pérenniser la production des SE et garantir un approvisionnement durable, il est nécessaire de faire en sorte que, d'une part, les écosystèmes soient réhabilités et conservés et, d'autre part, les activités économiques et les choix de gestion des terres et des ressources naturelles soient en phase avec une logique de production des SE. Toutefois, le problème est que ces bénéfices sont utilisés gratuitement tandis que leur production nécessite des investissements et des coûts importants. À cet effet, les PSE visent à rendre visibles les bénéfices issus de l'usage des SE à travers leur évaluation et leur échange moyennant un prix établi au sein de nouveaux marchés. L'idée étant d'utiliser les fonds issus des paiements pour financer la conservation en incitant les agents économiques à adopter des comportements et des techniques de gestion qui permettent d'améliorer la production des SE. L'application des PSE concerne une variété de ressources et d'écosystèmes en se basant sur une conception et des fondements communs.

\section{LES PSE: CONCEPT, FONDEMENTS ET APPLICATION}

Un PSE est défini comme étant une transaction volontaire où une demande (au moins un demandeur) rencontre, de façon volontaire, une offre (au moins un offreur) au sujet de la production d'un service environnemental spécifique et

Tableau 1. Catégories, description et exemples des services écosystémiques (MEA, 2005).

Table 1. Categories, description and examples of ecosystem services.

\begin{tabular}{|c|c|c|}
\hline Catégorie & Description & Exemples \\
\hline $\begin{array}{l}\text { Services } \\
\text { d'approvisionnement }\end{array}$ & $\begin{array}{l}\text { Produits prélevés directement } \\
\text { à partir des écosystèmes }\end{array}$ & $\begin{array}{l}\text { Aliments } \\
\text { Eau douce } \\
\text { Fibres et combustibles } \\
\text { Ressources génétiques } \\
\text { Produits biochimiques } \\
\text { Matériaux de construction }\end{array}$ \\
\hline $\begin{array}{l}\text { Services } \\
\text { de régulation }\end{array}$ & $\begin{array}{l}\text { Bénéfices obtenus de la } \\
\text { régulation assurée par le } \\
\text { fonctionnement des } \\
\text { écosystèmes (naturel ou } \\
\text { amélioré par l'action de } \\
\text { l'homme) }\end{array}$ & $\begin{array}{l}\text { Régulation du climat } \\
\text { Régulation hydrologique (protection contre les } \\
\text { inondations, protection contre l'érosion, qualité } \\
\text { de l'eau, infiltration de l'eau en profondeur, etc.) } \\
\text { Protection contre la désertification } \\
\text { Protection contre la pollution }\end{array}$ \\
\hline Services culturels & $\begin{array}{l}\text { Bénéfices matériels et } \\
\text { immatériels obtenus des } \\
\text { écosystèmes }\end{array}$ & $\begin{array}{l}\text { Paysages } \\
\text { Récréation } \\
\text { Services pédagogiques } \\
\text { Services spirituels/religieux } \\
\text { Connaissances et savoir-faire traditionnels }\end{array}$ \\
\hline $\begin{array}{l}\text { Services } \\
\text { de support }\end{array}$ & $\begin{array}{l}\text { Services nécessaires à la } \\
\text { production de tous les autres } \\
\text { services écosystémiques }\end{array}$ & $\begin{array}{l}\text { Formation des sols } \\
\text { Cycle de l'eau } \\
\text { Photosynthèse } \\
\text { Cycle des aliments }\end{array}$ \\
\hline
\end{tabular}


mesurable (WUNDER, 2005). La relation a lieu selon un contrat définissant la valeur des paiements, leurs échéances et les conditions requises pour qu'ils soient effectués (conditionnalité).

L'objectif des PSE est d'inciter à un changement de comportement en ce qui concerne l'usage, la gestion des écosystèmes et l'allocation des terres. À travers la mise en place de paiements, le fonctionnement des PSE vise à diminuer la pression sur les écosystèmes, à réhabiliter leurs principales fonctions écologiques et à améliorer, par conséquent, la production des SE (Figure 1). Ceci se base sur une logique économique qui admet que les utilisateurs d'un écosystème (pour le pâturage, la coupe de bois, le rejet de polluants, etc.) continuent à dégrader parce que cela leur permet de gagner un revenu élevé, à court terme, comparativement au cas où ils respectent des restrictions imposées par la conservation. Ces dernières ont un coût souvent lourd et difficile à supporter. Par exemple, conserver un pâturage collectif par sa mise en défens réduit le revenu des éleveurs, qui sont dans ce cas contraints à réduire la taille de leur cheptel ou à acheter des aliments de substitution pour leur cheptel, etc. Ce sont des coûts qui n'incitent pas à conserver les écosystèmes en absence de PSE (Figure 2). Pour que la conservation ait lieu de façon volontaire, il faudrait au moins compenser la perte de revenu à laquelle la conservation donne lieu. La logique économique des PSE propose à ce que cette diminution de revenus soit supportée par des paiements consentis par les autres agents économiques qui tirent profit de la conservation. Ces derniers supportent des coûts lorsque les écosystèmes sont dégradés parce que les SE dont ils bénéficient diminuent, ce qui les oblige à supporter des coûts que la conservation des écosystèmes en amont peut leur épargner. Par exemple, la dégradation des forêts ou du couvert végétal des parcours en amont d'un bassin versant réduit la production des $\mathrm{SE}$ de régulation hydrologique, de régulation de l'érosion, de la biodiversité, de séquestration du dioxyde de carbone, etc., ce qui pousse les utilisateurs de l'eau du barrage à investir pour filtrer l'eau, à supporter la rareté de l'eau causée par l'envasement du barrage, les chasseurs de gibier à dépenser plus pour chercher des endroits lointains pour chasser ou les citadins à souffrir de maladies causées par la pollution. Pour éviter ces coûts, les agents bénéficiaires peuvent consentir des paiements, en guise de rémunération des efforts fournis pour la conservation. Ces paiements permettent, ainsi, d'inciter les utilisateurs des écosystèmes en amont à conserver les écosystèmes.

La mise en œuvre des PSE connaît une propagation et un développement rapides à travers le monde. L'amélioration de la séquestration du carbone par les forêts, la conservation de la biodiversité, la mise en valeur des aménités paysagères par l'écotourisme et la récréation dans les aires protégées ainsi que l'amélioration de la disponibilité et de la qualité de l'eau par la conservation des bassins versants sont les objectifs de leurs principales applications (BENNETT et al., 2013). La production des SE groupés en bouquets de services est également une modalité qui a émergé depuis quelques années.

Les applications ne satisfont pas toutes nécessairement les cinq critères de définition des PSE. Il y a très peu d'exemples de PSE volontaires (BENNETT et al., 2013; LAURENS et al., 2011). La majorité sont des PSE organisés par les gouvernements qui paient les propriétaires de terres privées (forêts privées, pâturages, terres agricoles, etc.) pour maintenir ou augmenter la production des SE désirés. Une troisième catégorie, moins répandue que les PSE gouvernementaux, prend la forme de marchés mis en place et organisés par les États tout en laissant les transactions avoir lieu de façon volontaire entre les agents économiques (LAURENS et al., 2011). Cette dernière catégorie est généralement régie par deux principaux types de règles. Le premier est un système dit Cap and Trade, où les échanges ont lieu selon des limites minimales ou maximales fixées pour la fourniture des SE. Le deuxième est un système qui laisse l'offre et la demande libres de s'entendre sur ce qui les arrange mutuellement.

Globalement, les PSE apportent des solutions pour de sérieux problèmes environnementaux. Parmi ces problèmes, il y a celui de la conservation des bassins versants et la conservation des ressources en eau, que l'on aborde dans la section suivante.

\section{LES PSE APPLIQUÉS À LA CONSERVATION DES BASSINS VERSANTS}

Les bassins versants représentent un objet privilégié pour la mise en œuvre des PSE, du fait qu'ils rassemblent une variété d'écosystèmes offrant des possibilités d'action en fonction des problèmes environnementaux détectés et du contexte socioéconomique en présence (MURADIAN et al., 2010). Les programmes de PSE dédiés à la conservation des ressources en eau à l'échelle des bassins versants ne cessent d'augmenter sur les cinq continents du globe (Tableau 2).

Les PSE de conservation des bassins versants sont souvent structurés autour de l'amélioration, ou du maintien, de la production des SE de la régulation hydrologique. De par la multifonctionnalité des écosystèmes, cette amélioration (ou maintien) peut être réalisée par plusieurs types d'interventions. Les voies empruntées fréquemment se basent sur la conservation et la réhabilitation des forêts (boisement et reboisement) et sur l'amélioration des pratiques de gestion en agriculture dans la partie amont des bassins versants. 


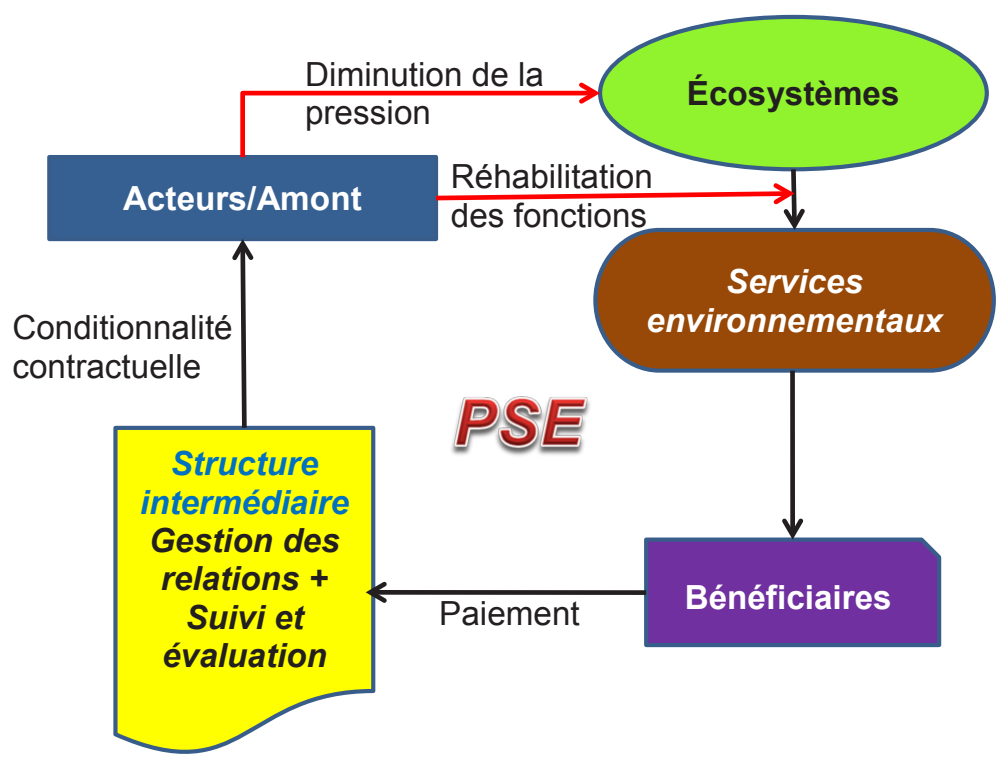

Figure 1. Schéma simplifié de fonctionnement d'un paiement pour services environnementaux (PSE).

Simplified scheme of a payment for environmental services (PES)

functioning.

$\begin{array}{ccc}\text { Sans } & \text { Conservation } & \text { Conservation } \\ \text { conservation } & \text { SANS PSE } & \underline{\text { AVEC PSE }}\end{array}$

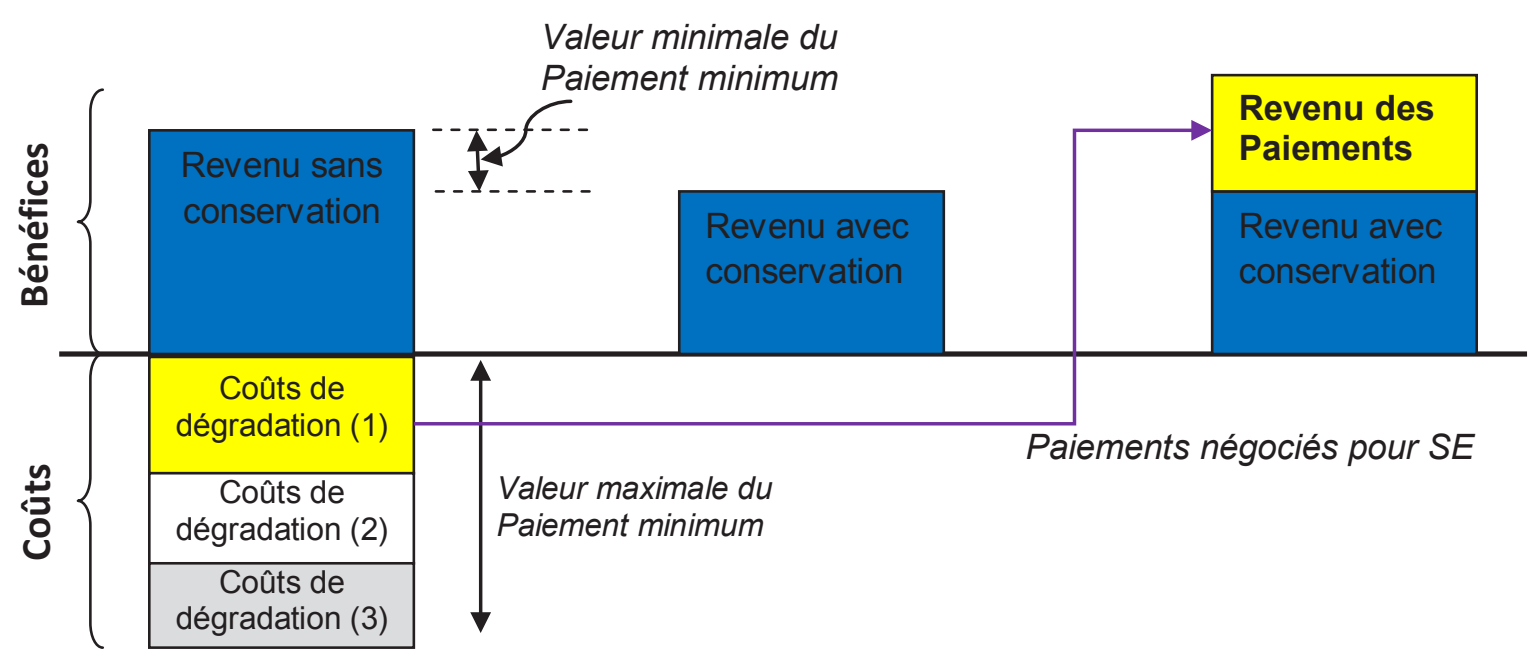

Figure 2. La logique économique d'un paiement pour services environnementaux (PSE) (adapté d'ENGEL et al., 2008). Les (1), (2) et (3) illustrent les coûts produits par la dégradation d'un bassin versant :(1) par l'altération des SE liés à l'eau (altération de la qualité de l'eau, diminution de sa disponibilité, etc.); (2) par la dégradation de la biodiversité; (3) par la diminution de la capacité de l'écosystème à stocker le dioxyde de carbone. The economic logic of a payment for environmental services(PES) (adapted from ENGEL et al., 2008). 
Tableau 2. Nombre de paiement pour services environnementaux (PSE) appliqués à la conservation des bassins versants dans le monde (BENNETT et al., 2013).

Table 2. Number of worldwide payment for environmental services (PES) applications to watershed conservation.

\begin{tabular}{|c|c|c|c|c|}
\hline \multirow[b]{2}{*}{ Continents } & \multicolumn{2}{|c|}{ Nombre de PSE par stade } & \multirow[b]{2}{*}{ Principaux pays $^{a}$} & \multirow[b]{2}{*}{ Total } \\
\hline & PSE actifs & $\begin{array}{c}\text { PSE en cours } \\
\text { de montage }\end{array}$ & & \\
\hline Afrique & 6 & 10 & $\begin{array}{c}\text { Afrique du Sud (3), Kenya (4), } \\
\text { Tanzanie (3) }\end{array}$ & 16 \\
\hline Europe & 15 & 3 & $\begin{array}{c}\text { Angleterre (4), Allemagne (4), } \\
\text { France (3), Espagne (3) }\end{array}$ & 18 \\
\hline Amérique latine & 28 & 8 & $\begin{array}{c}\text { Costa Rica (2), Équateur (12), Brésil } \\
\text { (7), Bolivie (6), Mexique (3) }\end{array}$ & 36 \\
\hline Amérique du Nord & 68 & 18 & USA (85), Canada (1) & 86 \\
\hline Australie et Asie & 87 & 36 & $\begin{array}{c}\text { Chine (80), Philippines (9), Japon (4), } \\
\text { Indonésie (14) }\end{array}$ & 123 \\
\hline Total & 204 & 75 & - & 279 \\
\hline
\end{tabular}

${ }^{a}$ Les chiffres entre parenthèse indiquent le nombre de projet dans les principaux pays abritant un nombre relativement élevé de PSE dans chaque continent, les autres pays ne sont pas mentionnés.

Les PSE mis en ouvre à travers le monde ont permis, seulement en 2011, la mobilisation d'un montant global de 8,17 milliards de dollars et la conservation d'une superficie globale de 195 millions d'hectares dans les bassins versants (BENNETT et al., 2013). En Afrique, six programmes sont actifs et ont permis, en 2011, la mobilisation de plus de 109,3 millions de dollars pour conserver plus de 161000 ha (BENNETT et al., 2013). Le pays d'Afrique où on dénombre le plus de PSE actifs est l'Afrique du Sud (Tableau 2).

\section{LE CAS DE LA GESTION ET DE LA CONSERVATION DES BASSINS VERSANTS AU MAROC}

L'un des défis majeurs du Maroc engagé sur la voie du développement durable concerne la conservation des bassins versants. C'est l'échelle adoptée, depuis les années 1980, pour la planification, l'aménagement et la gestion des ressources en eau et en sol. Depuis 1996, le Maroc s'est doté d'un Plan National d'Aménagement des Bassins Versants (PNABV) qui vise, en plus de la satisfaction de la demande en eau, à améliorer la conservation des ressources en eau et en sol. Il propose, à cet effet, une conservation intégrée des ressources selon un ordre de priorités et un montage financier nouveaux. Contrairement aux politiques passées orientées vers un objectif de conservation des investissements hydroagricoles en aval des barrages, le PNABV propose de prioriser les interventions de l'État en se basant sur des critères qui concernent aussi bien l'amont que l'aval.
Les études réalisées durant la phase préparatoire du PNABV ont concerné 15 millions d'hectares et ont montré que $75 \%$ des superficies étudiées courent un risque élevé d'érosion hydrique. Les pertes annuelles cumulées en sols sont estimées à 100 millions de tonnes occasionnant une perte annuelle en capacité de stockage de 50 millions de mètres cubes (HCEFLCD, 2011). En termes financiers, les pertes totales dues à l'érosion sont évaluées à 10 milliards de dirhams (MAD). Ceci a révélé la nécessité de conserver les écosystèmes en amont des bassins versants pour un objectif nouveau qui consiste à éviter ou à réduire les coûts économiques de la dégradation. Un rythme de conservation de $75000 \mathrm{ha} \cdot \mathrm{an}^{-1}$ a été proposé pour une durée de 20 ans.

En pratique, après près de 20 années de mise en œuvre du PNABV, les problèmes de l'érosion, d'altération, de la qualité de l'eau et des capacités de stockage demeurent au niveau des principales retenues du pays. Les difficultés de financement des aménagements restent insurmontables (OUBALKACE, 2007). Aussi, les choix techniques effectués restent subjectifs et peu efficients en absence de connaissances scientifiques et d'évaluations économiques qui renseignent sur les impacts et orientent les investissements. Cependant, les principales dispositions du PNABV, telles qu'elles ont été formulées au départ, recommandent des mesures très semblables à ce qui fait ailleurs le succès des programmes de PSE. Cependant, le manque d'efficacité peut éventuellement être expliqué par le financement unilatéral par l'État et par l'absence d'engagement volontaire et contractuel des agents économiques en amont et en aval des bassins versants. Les PSE présentent cet avantage et permettent d'agir d'une autre manière. A cet effet, et dans l'absence d'un modèle de PSE standard et valable partout, il 
convient d'évaluer ce que pourront être les apports d'un tel mécanisme ainsi que la forme qu'il pourra prendre au Maroc, pour engager une coopération multipartite et offrir la flexibilité technique et financière permettant d'intégrer les spécificités socioéconomiques des différentes régions du pays (KELSEY JACK et al., 2008; WUNDER, 2006).

\section{LA FORME ET LES APPORTS POTENTIELS DES PSE AU MAROC}

La mise en œuvre des PSE nécessite le choix d'une forme appropriée. Les trois formes de PSE qui existent aujourd'hui (voir section 3) présentent chacune des avantages et des inconvénients pour une future application au Maroc. Les PSE volontaires offrent plus de flexibilité et de liberté de choix des méthodes de conservation, mais leur application est difficile sans supporter des coûts d'information qui risquent d'être très élevés (MILDER et al., 2010). L'absence d'expériences préalables de PSE et le manque d'information par rapport à leur fonctionnement et leur logique sont des handicaps (WUNDER, 2013). Les PSE volontaires régulés par l'État présentent également les mêmes inconvénients et risquent de ne pas connaître un développement qui puisse satisfaire l'énorme besoin de conservation à court et moyen termes. Enfin, les PSE régulés et financés par l'État présentent un risque quant à l'insuffisance des budgets et leur irrégularité, déjà ressentie aujourd'hui.

La forme appropriée semble être, finalement, un mélange entre les deux types de PSE régulés par l'État. Ce dernier aura la mission de mettre en place la vision et les règles du jeu et de participer partiellement au financement, en combinaison avec les paiements de bénéficiaires. La concentration des fonds publics dédiés à la conservation (paiements, budgets étatiques, aides, etc.) dans un fonds (national ou régional) dédié au financement des PSE est susceptible d'offrir une flexibilité et une régularité financières. Toutefois, cette forme devra obligatoirement être appuyée par la mise en place d'un cadre politique et réglementaire clair qui permet de sécuriser les droits des agents économiques (payeurs et producteurs de SE).

En ce qui concerne les apports potentiels des PSE, leur mise en ouvre à travers le monde pour la conservation des bassins versants montre qu'ils constituent une alternative prometteuse du fait de leur supériorité par rapport aux outils classiques de conservation basés sur une logique de développement intégré (LAURENS et al., 2011; PAGIOLA et al., 2005; PASCUAL et al., 2010) et par rapport aux outils réglementaires insistant sur le contrôle et la dissuasion (HUBER et al., 1998). Ils sont plus efficaces et permettent d'améliorer le financement de la conservation. En s'appuyant sur une approche de services environnementaux, les PSE basent la conservation sur des objectifs quantifiés tirés des évaluations et des données de suivi qui permettraient de mieux orienter les décisions de conservation.

Les PSE représentent, également, une occasion pour améliorer le montage institutionnel actuel au niveau des bassins versants en impliquant davantage, de façon ciblée en fonction des objectifs spécifiques de la conservation, des acteurs privés et la population. Ceci constitue un avantage pour dépasser la prise de décision sectorielle et centralisée, adoptée pour la conservation de la plupart des ressources naturelles, vers une prise de décision flexible, adaptée aux réalités disparates des contextes locaux et qui prend en compte les préférences des acteurs concernés.

Par ailleurs, les avantages que promet la mise en œuvre des PSE au Maroc restent tributaires d'une volonté politique et d'une mobilisation synergique de tous les acteurs. Le pays présente des spécificités qui peuvent façonner d'une manière particulière une mise en ouvre future des PSE au Maroc.

\section{LES SPÉCIFICITÉS LOCALES: AVANTAGES ET LIMITES POUR LA MISE EN GEUVRE DES PSE}

La mise en œuvre des PSE au Maroc sera confrontée aux spécificités des modes de gestion des terres en relation avec la conservation des écosystèmes. L'existence, au Maroc, de près de 15 millions d'hectares de terres collectives et de plus de 51 millions d'hectares de parcours forestiers et hors forestiers est une spécificité importante à laquelle les PSE seront confrontés au niveau du pays. Ce genre de contextes de propriété collective est évité dans la mise en œuvre des PSE en raison des coûts élevés que nécessite leur mise en place (coûts de transaction nécessaires pour organiser la population, pour négocier les contrats, etc.). Les conditions privilégiées étant une définition claire des droits de propriété et un contrôle fort des fournisseurs de SE sur les terres (ENGEL et PALMER, 2008).

Toutefois, la dégradation des forêts et du couvert végétal des parcours, dont l'usage est collectif, est un problème sérieux. La conservation des bassins versants ne peut pas se passer de la résolution de ce problème, qui a de lourdes conséquences sur la régulation hydrologique dans toutes ses dimensions. De ce fait, la mise en ouvre des PSE au Maroc doit être adaptée à ce contexte. En fait, le problème des terres collectives en relation avec la mise en œuvre des PSE n'est pas seulement un problème de statut juridique, mais une question de déploiement de ces 
droits pour définir les règles de gestion et d'usage. Le droit de propriété collective, attribué aux communautés villageoises ou à des tribus au Maroc, est soumis à une tutelle du Ministère de l'Intérieur. La jouissance est collective et la gestion est soumise à un processus délibératif interne à la tribu et/ou la Jmaâa (une sorte de conseil de village). L'existence d'une organisation sociale ancestrale pour le partage des droits d'usage et la gestion des espaces collectifs représente, à cet effet, un avantage qui permet de réduire très significativement les coûts de transaction liés à la mise en œuvre des PSE. La possibilité de coopération entre les collectivistes faciliterait la réussite des engagements en termes de gestion conservatoire. Ceci permettrait de réduire les coûts de transactions nécessaires à la mise en place des PSE et faciliterait leur implantation au Maroc, où les pâturages et les forêts sont soumis à l'usage collectif des communautés et des tribus.

Par ailleurs, il existe des cas où l'exploitation collective peut poser un problème et où plus d'efforts devrait avoir lieu en termes de conception des PSE. Il s'agit des tribus qui s'étendent sur des surfaces vastes et qui englobent une population nombreuse. Dans ce genre de contexte, les structures traditionnelles de gestion sont affaiblies et n'ont pas de pouvoir de décision, ce qui risque d'augmenter les coûts de transactions liés à l'organisation de la population et à son information. Le même cas pourrait se poser dans des contextes où existent des formes d'organisation modernes (associations, coopératives, etc.) dont la légitimité et la représentativité sont remises en question par les populations de collectivistes. Cependant, la modélisation des préférences individuelles et collectives des populations peut aider à mieux cibler les actions et à renseigner sur les attributs du PSE susceptibles de garantir une large adhésion.

La difficulté à recenser les ayants droit au sein des forêts et pâturages collectifs ainsi que de distinguer entre un ayant droit et un usager, sont des difficultés additionnelles au sujet desquelles une réflexion nationale devra être engagée.

D'autres limites peuvent influencer la mise en œuvre des PSE. Il s'agit, notamment, de : i) l'absence d'une culture de paiement par les bénéficiaires des SE pour le financement des actions de conservation qui ont toujours été financées par l'État; ii) la relation de l'État avec les populations, qui reste ancrée par des antécédents historiques qui varient d'un contexte à l'autre selon la réussite ou l'échec des expériences de conservation passées; iii) des contraintes techniques relatives à l'absence de données et de connaissances scientifiques permettant de faciliter la mesure des SE produits; iv) l'habitude des administrations sectorielles à agir unilatéralement et la difficulté à élargir la coopération vers un système de gouvernance plus flexible et synergique; $v$ ) la sensibilité politique des paiements lorsqu'il s'agit d'un paiement additionnel ou d'une ressource particulière telle que l'eau d'irrigation.

\section{CONCLUSION}

L'utilisation des PSE pour améliorer la conservation des bassins versants représente, au Maroc, une opportunité pour améliorer le financement de la conservation et donner lieu à une nouvelle vision basée sur l'efficience et l'efficacité des investissements. Les différents attributs de l'eau, comme la disponibilité et la qualité, ont des chances d'être améliorés à travers la mise en œuvre de PSE. Ces derniers permettraient également d'améliorer la conservation des équilibres écologiques et la durabilité des activités économiques au sein des bassins versants. Toutefois, les PSE ne sont pas une solution à tous les problèmes. Leur application doit être justifiée en termes de coûts et de bénéfices et accompagnée par une amélioration aux niveaux institutionnel, organisationnel et réglementaire. Aussi, l'intérêt des PSE doit être examiné en fonction de leur capacité spécifique à apporter des solutions eu égard aux spécificités locales. La conception et la mise en ouvre selon un schéma régulé par l'État ne doivent pas signifier, dans la pratique, une obligation des acteurs concernés ou leur association forcée au paiement ou au respect des pratiques identifiées comme nécessaires à l'amélioration de la régulation hydrologique. Le caractère volontaire reste un gage de réussite. En ce sens, il convient d'investir dans la compréhension des préférences des populations et de chercher une adéquation entre les choix de gestion et de conservation avec ce que les agents économiques jugent opportun en fonction de leurs perceptions et contraintes (sociales, économiques, culturelles, etc.). Ceci permettrait également de résoudre les problèmes prévisibles de la propriété collective et aiderait à formuler des PSE avec des mesures adaptées à la volonté des populations et des niveaux de paiements raisonnables.

$\mathrm{Au}$ Maroc, la plus importante limite à l'implantation des PSE est la faiblesse des connaissances et des données qui permettent de comprendre les interactions au sein et entre les différents écosystèmes que réunissent les bassins versants. Le développement de la recherche doit accompagner le développement des PSE. Les enseignements des expériences passées (plans, programmes, projets, etc.) ainsi que les expériences de conservation traditionnelle des ressources naturelles (gestion sociale de l'eau, agdals [des espaces pastoraux soumis à une mise en défens volontaire traditionnelle et très ancienne chez les populations Amazigh du Haut et du Moyen Atlas marocains] forestiers et pastoraux, etc.) peuvent avoir des apports non négligeables.

\section{REMERCIEMENTS}

Nous tenons à remercier vivement les deux évaluateurs anonymes qui ont pris le soin de lire l'article et de nous aider 
par leurs remarques et commentaires à améliorer la première version. Nous remercions également le professeur M.R. Doukkali de l'Institut Agronomique et Vétérinaire Hassan II et C. Lejars du CIRAD pour leurs conseils et leur appui.

\section{RÉFÉRENCES BIBLIOGRAPHIQUES}

BENNETT G., N. CARROLL et K. HAMILTON (2013). Charting new waters: State of watershed payments 2012. Forest Trends, Washington, DC, États-Unis, 98 p.

DAILY G.C. (1997). Nature's services: Societal dependence on natural ecosystems. Island Press, Washington, DC, ÉtatsUnis, $412 \mathrm{p}$.

ENGEL S., S.PAGIOLA et S. WUNDER (2008). Designing payments for environmental services in theory and practice: An overview of the issues. Ecol. Econ., 65, 663-674.

ENGEL S. et C. PALMER (2008). Payments for environmental services as an alternative to logging under weak property rights: The case of Indonesia. Ecol. Econ., 65, 799-809.

EROL A. et T.O. RANDHIR (2013). Watershed ecosystem modeling of land-use impacts on water quality. Ecol. Model., 270, 54-63.

Food and Agriculture Organization of the United Nations (FAO) (2007). La situation mondiale de l'alimentation et de l'agriculture: payer les agriculteurs pour les services environnementaux. Rome, Italie, 259 p.

FIQUEPRON J., S. GARCIA et A. STENGER (2013). Land use impact on water quality: Valuing forest services in terms of the water supply sector. J. Environ. Manage., 126, 113-121.

HAUT COMMISSARIAT AUX EAUX ET FORÊTS ET À LA LUTTE CONTRE LA DÉSERTIFICATION (HCEFLCD) (2011). Plan national d'aménagement des bassins versants : résumé et conclusions de rapport de synthèse. Maroc, $53 \mathrm{p}$.

HUBER, R.M., J. RUITENBEEK, et R.S. DA MOTTA (1998). Market based instruments for environmental policymaking in Latin America and the Caribbean: Lessons from eleven countries. World Bank Discussion Paper n ${ }^{\circ} 381$, Washington, DC, États-Unis, 92 p.

KELSEY JACK B., C. KOUSKY et K.R.E. SIMS (2008). Designing payments for ecosystem services: Lessons from previous experience with incentive-based mechanisms. Proc. Natl. Acad. Sci (USA), 105 (28), 9465-9470.
LAURENS Y., T. LEMENAGER et S. AOUBID (2011). Les paiements pour les services environnementaux. De la théorie à la mise en auvre, quelles perspectives dans les pays en développement. Agence Française de Développement (AFD), Â savoir nº 07, France, 218 p.

MILDER J.C., S.J. SCHERR et C. BRACER (2010). Trends and future potential for payment for ecosystem services to alleviate rural poverty in developing countries. Ecology and Society, 15 (2), 4. URL: http://www.ecologyandsociety. org/vol15/iss $2 /$ art $4 /$

MILLENNIUM ECOSYSTEM ASSESSMENT (MEA) (2005). Ecosystems and human well-being: Current state and trends. Island Press, Washington, DC, États-Unis, 155 p.

MURADIAN R., E. CORBERA, U. PASCUAL, N. KOSOY et P.H. MAY (2010). Reconciling theory and practice: An alternative conceptual framework for understanding payments for environmental services. Ecol. Econ., 69, 1202-1208.

OUBALKACE M. (2007). Stratégie méditerranéenne pour le développement durable. Suivi des progrès dans le domaine de l'eau et promotion de politiques de gestion de la demande. Commission Méditerranéenne du Développement Durable, Ministère de l'Aménagement du Territoire, de l'Eau et de l'Environnement, Maroc, 120 p.

PAGIOLA S., A. ARCENAS, et G. PLATAIS (2005). Can payments for environmental services help reduce poverty? An exploration of the issues and the evidence to date. World Dev., 33 (2), 237-253.

PASCUAL U., R. MURADIAN, L.C. RODRÍGUEZ et A. DURAIAPPAH (2010). Exploring the links between equity and efficiency in payments for environmental services: A conceptual approach. Ecol. Econ., 69, 12371244.

WUNDER S. (2005). Payments for environmental services: Some nuts and bolts. Center for International Forestry Research (CIFOR), Occasional paper n 42, Bogor, Indonésie, 32 p.

WUNDER S. (2006). Are direct payments for environmental services spelling doom for sustainable forest management in the Tropics? Ecology and Society, 11(2), 23. URL: http://www.ecologyandsociety.org/vol11/iss2/art23/

WUNDER S. (2013). When payments for environmental services will work for conservation. Center for International Forestry Research (CIFOR), Rio de Janeiro, Brésil, 8 p. 